\title{
Probing the indistinguishability of single photons generated by Rydberg atomic ensembles
}

\author{
Auxiliadora Padrón-Brito $\mathbb{C}^{1},{ }^{1}$ Jan Lowinski $\odot,{ }^{1}$ Pau Farrera, ${ }^{1,2}$ Klara Theophilo, ${ }^{1}$ and Hugues de Riedmatten ${ }^{1,3}$ \\ ${ }^{1}$ ICFO-Institut de Ciències Fotòniques, Barcelona Institute of Science and Technology, 08860 Castelldefels, Barcelona, Spain \\ ${ }^{2}$ Max-Planck-Institut für Quantenoptik, 85748 Garching, Germany \\ ${ }^{3}$ ICREA-Institució Catalana de Recerca i Estudis Avaçats, 08015 Barcelona, Spain
}

(Received 9 November 2020; revised 21 June 2021; accepted 3 September 2021; published 29 September 2021)

\begin{abstract}
We investigate the indistinguishability of single photons retrieved from collective Rydberg excitations in cold atomic ensembles. The Rydberg spin waves are created either by off-resonant two-photon excitation to the Rydberg state or by Rydberg electromagnetically induced transparency. To assess the indistinguishability of the generated single photons, we perform Hong-Ou-Mandel experiments between the single photons and weak coherent states of light. We analyze the indistinguishability as a function of the detection window, and we infer a high value of indistinguishability going from $89 \%$ for the full waveform to $98 \%$ for small detection windows, for the case of photons generated by off-resonant excitation. In the same way, we also investigate the indistinguishability of single photons generated by Rydberg EIT, showing values lower than those corresponding to single photons generated by off-resonant excitation. These results are relevant for the use of Rydberg atoms as quantum network nodes.
\end{abstract}

DOI: 10.1103/PhysRevResearch.3.033287

\section{INTRODUCTION}

Collective Rydberg atomic excitations are an interesting system for realizing quantum network nodes [1], due to the strong long-range interaction between two Rydberg atoms [2]. These collective excitations constitute a nonlinear medium at the single-photon level with a stronger light-matter interaction than that of a single atom and therefore provide the capability to generate quasideterministic light-matter entanglement and to perform quantum processing between stored qubits [3] or between single photons [4-6]. The use of electromagnetically induced transparency (EIT) with Rydberg atoms provides an efficient way to map photons onto Rydberg states [7,8], therefore enabling photon-photon interactions. Peyronel et al. [9] first demonstrated nonlinearity at the single-photon level under Rydberg EIT conditions in 2012, and since then this method has been exploited to generate single photons [10], realize single-photon switches and transistors [11-13], develop photon-photon gates with weak coherent states [14], and demonstrate interaction between collective Rydberg excitations stored in separate atomic ensembles [15], among other work. In parallel, other experiments used off-resonant (OR) two-photon excitation to a Rydberg state, where the population of the intermediate state can be neglected, to generate single photons [16,17], perform deterministic generation of ground-state spin waves $[18,19]$, and pursue light-matter entanglement $[20,21]$.

Published by the American Physical Society under the terms of the Creative Commons Attribution 4.0 International license. Further distribution of this work must maintain attribution to the author(s) and the published article's title, journal citation, and DOI.
Future quantum networks would rely on the capability of generating entanglement between remote nodes. In most of the proposed quantum repeater architectures, including the ones based on Rydberg interactions [22,23], the remote nodes are entangled by a measurement-induced process which involves interference of single photons emitted by the nodes and a Bell state measurement. For this process to be successful, the single photons must be indistinguishable, which means the information about the path is erased. The standard method of probing the photons indistinguishability is Hong-Ou-Mandel (HOM) interference [24], in which two single photons impinge on a beamsplitter (BS) and if indistinguishable exit bunched in the same output mode. So far, only a few experiments have probed the indistinguishability of single photons emitted by Rydberg spin waves [20,25,26]. In the work carried out by Li et al. (2013) the photon indistinguishability was assessed by performing the HOM experiment between the single photons emitted by the Rydberg spin wave and a weak coherent state [20]. Craddock et al. (2019) in a very challenging experiment interfered Rydberg photons with photons emitted by a single ion [25] proving indistinguishability between them. These two experiments, however, investigated only a small part of the temporal waveform of the probed single photons. While this strategy allows observing high-visibility HOM interference, it reduces the available two-photon coincidences by a factor $\sim(\Delta t / T)^{2}$, where $\Delta t$ is the detection window considered and $T$ is the total photon waveform duration, thus reducing the projected entanglement generation rate. The work recently carried out by Ornelas-Huerta et al. [26] showed high indistinguishability by interfering two consecutive single photons emitted by the same Rydberg ensemble. This work examined the whole pulse duration; however, similar to the previous ones, it investigated only single photons generated by off-resonant two-photon 
(a)

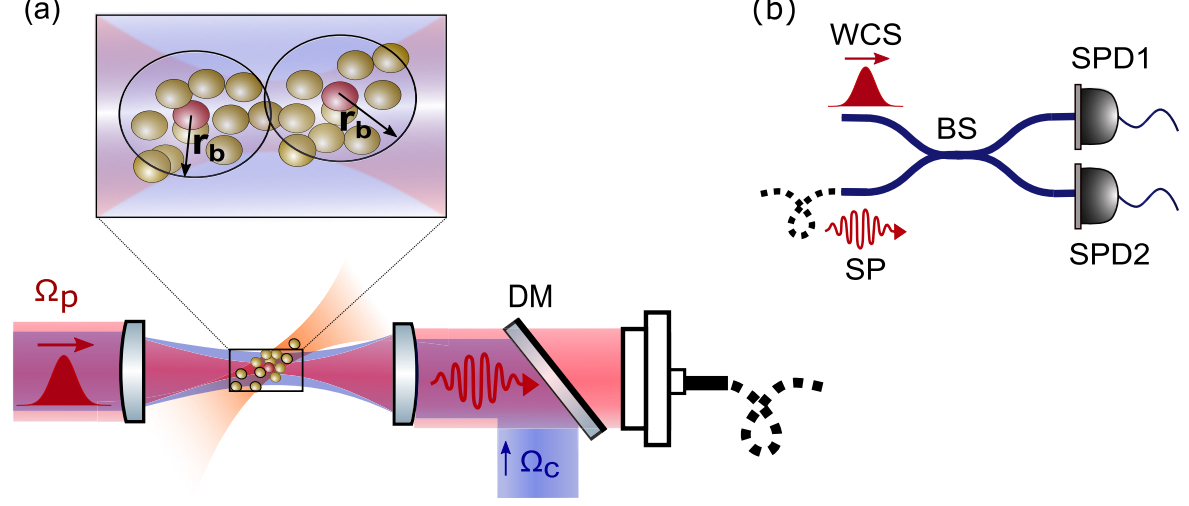

(c)

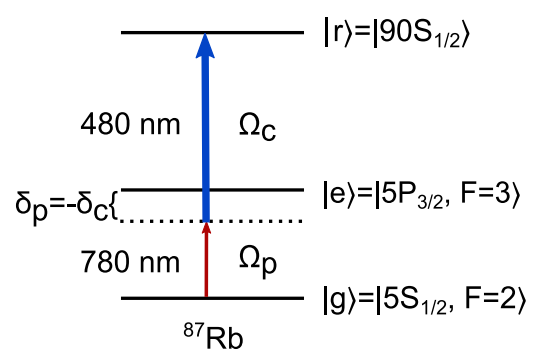

FIG. 1. (a) Schematic representation of the experimental setup. An input probe pulse (in red) is sent to the experiment with a spatial distribution given by the probe beam (shadowed red). The control beam (in blue) is counterpropagated with the probe, and both are focused in the center of the atomic cloud by two aspheric lenses. (b) The emitted single photon (SP) interferes with a WCS pulse in a HOM experiment, consisting of a fiber-based beamsplitter and two single-photon detectors SPD1 and SPD2. (c) Level scheme and transitions used in the experiment, where $\delta_{p}$ and $\delta_{c}$ are the detuning of the probe and control beams with regard to the intermediate level. $\Omega_{p}$ : probe Rabi frequency, $\Omega_{c}$ : control Rabi frequency, DM: dichroic mirror.

excitation. The indistinguishability of single photons generated by Rydberg EIT, and the differences between both processes, has to our knowledge not been experimentally investigated so far.

In this paper, we investigate the indistinguishability of photons retrieved from collective Rydberg excitations in a cold atomic ensemble. The Rydberg spin waves are created either by OR two-photon excitation to the Rydberg state or by Rydberg EIT. To assess the indistinguishability of the generated single photons, we perform HOM experiments between the single photons and weak coherent states of light and observe nonclassical HOM visibilities. We study the overlap between the single photons and weak coherent states as a function of the detection window, and we find that the photons generated under Rydberg EIT conditions show lower overlap than the ones generated by OR excitation. Finally, we discuss possible reasons for the lower indistinguishability observed for EIT photons.

\section{EXPERIMENT}

The experimental setup scheme and the relevant atomic levels are shown in Fig. 1. A cold cloud of ${ }^{87} \mathrm{Rb}$ atoms is loaded in a dipole trap, with an atomic peak density of $\sim 4 \times 10^{11} \mathrm{~cm}^{-3}$ and a transverse size of $34 \mu \mathrm{m}$. We start by loading a magneto-optical trap (MOT) for $2 \mathrm{~s}$, followed by a compression of the MOT and further cool down by optical molasses. The dipole trap of a focused laser beam of $852 \mathrm{~nm}$, which gives a trap depth of $\sim 0.3 \mathrm{mK}$, is switched on $500 \mathrm{~ms}$ after the beginning of the MOT and switched off during the excitation of the atoms to the Rydberg state, to avoid losing atoms and remove AC Stark shifts. This is done by modulating the dipole trap with a period of $5.6 \mu \mathrm{s}$, which gives $2.8 \mu \mathrm{s}$ to perform one experimental trial. Each trial consists of the generation of collective Rydberg excitations, by either OR or EIT excitation, and further retrieval of the single photons. We perform 37000 experimental trials at a rate of $178 \mathrm{kHz}$ during $200 \mathrm{~ms}$ within the trap's lifetime.

The atoms are initially prepared in the ground state $|g\rangle=\left|5 S_{1 / 2}, F=2\right\rangle$ and coupled to the excited state $|e\rangle=$ $\left|5 P_{3 / 2}, F^{\prime}=3\right\rangle$ by a weak probe field at $780 \mathrm{~nm}$. The probe beam is sent with an angle of $19^{\circ}$ with respect to the dipole trap beam [see Fig. 1(a)] and is focused to a beam waist of $w_{p} \approx 6.5 \mu \mathrm{m}$ in the center of the atomic medium, which has an optical depth (OD) $\sim 6$ for a resonant probe. The excited state $|e\rangle$ is coupled to the Rydberg state $|r\rangle=\left|90 S_{1 / 2}\right\rangle$ by means of a counterpropagating control beam at $479.4 \mathrm{~nm}$. The one-photon transition detunings are defined as $\delta_{p}=\omega_{g e}-\omega_{p}$ and $\delta_{c}=\omega_{e r}-\omega_{c}$, where $\omega_{p(c)}$ are the frequencies of the light fields and $\omega_{\text {ge(er })}$ the frequencies of the corresponding atomic transitions [see Fig. 1(c)]. For all experiments, the two-photon transition is sent on resonance so that $\delta_{c}+\delta_{p} \approx 0$.

To generate the single photons, we send both probe and control fields into the medium to create collective atomic Rydberg excitations that afterwards are retrieved in the form of a single photon. We use two different techniques: the first one is based on a two-photon OR excitation to the Rydberg state, and the second one utilizes stopped light of Rydberg EIT. In Fig. 2 we show the temporal profile of the input and output pulses for both cases. For the OR excitation, we send a probe pulse with average photon number $n_{\text {in }} \approx 10$ and a control pulse with Rabi frequency $\Omega_{c} \approx 2 \pi \times 6 \mathrm{MHz}$, both detuned
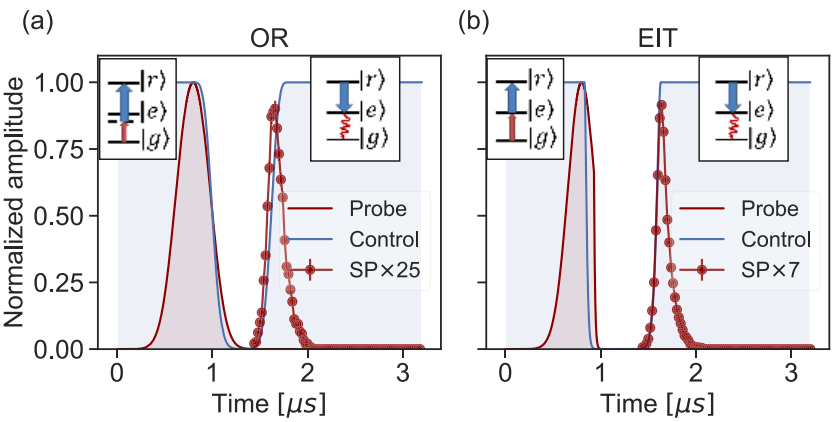

FIG. 2. Representation of the probe and control optical pulses used for the photon generation under (a) OR and (b) EIT conditions, normalized to their maximum value. SP shows the retrieved photon temporal distribution counts with respect to the input probe. 
from the $|e\rangle$ level by $\delta_{p}=-\delta_{c}=2 \pi \times 40 \mathrm{MHz}$. In the case of a fully blockaded medium, the two-photon absorption creates a single Rydberg collective excitation, also called a Rydberg spin wave. In our case, since the two-photon Rabi frequency $\Omega=\frac{\Omega_{p} \Omega_{c}}{4 \delta_{p}}$ is smaller than the combined laser linewidth $\gamma \approx$ $2 \pi \times 0.7 \mathrm{MHz}$, the blockade radius is determined by the latter and leads to $r_{b} \approx 16.9 \mu \mathrm{m}$, which is smaller than the effective medium length in the probe propagation direction ( $L \approx 104 \mu \mathrm{m}$ ). Therefore, more than one Rydberg excitation can be created in the interaction volume, but the dephasing of multiple Rydberg excitations during the storage time ensures that most of the time only one excitation can be retrieved collectively [16]. The imperfect single-photon emission, however, may cause lower photon indistinguishability [27]. Highly pure single-photon sources with collective Rydberg excitations have been recently demonstrated using a Rydberg state with $n=139$ that ensures a fully blockaded atomic medium [26]. Finally, the retrieval is performed by sending an on-resonant control pulse after a given storage time, which triggers the emission of the photons in the original mode of the probe.

The second method relies on stopping light propagating in the medium under the EIT conditions. For that, we send a weak probe pulse with average input photon number $n_{\text {in }} \approx$ 0.7 together with a control pulse with $\Omega_{c} \approx 2 \pi \times 6 \mathrm{MHz}$ into the atomic medium, both resonantly coupled to $|e\rangle$ level $\left(\delta_{c}=\delta_{p}=0\right)$. In the absence of Rydberg interactions, the control pulse creates a narrow transparency window, allowing the propagation of the probe photons as dark state polaritons with a reduced group velocity $v_{g} \ll c$ (slow light) [10,28,29]. The Rydberg dipole-dipole interactions, however, destroy the EIT transparency for two probe photons propagating within the blockade radius. As a consequence, strong scattering and absorption associated with a resonant two-level medium occurs. The blockade radius in this case is given by the EIT linewidth, which leads to $r_{b} \approx 13 \mu \mathrm{m}$. While the probe photons are inside the medium, the control field is turned off and the Rydberg polaritons are mapped onto stationary collective Rydberg excitations which can also undergo multicomponent dephasing. After a controllable storage time, the control field intensity is increased to its initial value, and the ideally single remaining dark state polariton regains its photonic characteristic and propagates until exiting the medium as a single photon.

The statistics of the photons emitted by the Rydberg spin waves is measured in an Hanbury-Brown and Twiss setup, composed of a fiber-based beamsplitter (BS) and two singlephoton detectors (SPDs). The photon arrival times at each SPD, together with trigger times for each trial, are saved in time-stamp files to perform analysis for different detection windows. The second-order autocorrelation function within a detection window of duration $\Delta t$ is then measured as

$$
g_{\Delta t}^{(2)}=\frac{P_{12}(\Delta t)}{P_{1}(\Delta t) P_{2}(\Delta t)},
$$

where $P_{12}(\Delta t)$ is the coincidence detection probability between both detectors and $P_{1(2)}(\Delta t)$ is the detection probability in the SPD1(2).

To probe the indistinguishability of the single photons, we use the HOM effect [24]. We perform an interference measurement between the photons emitted by the Rydberg spin waves (SP) and weak coherent state pulses (WCS) by sending the WCS to the other input port of the fiber-based BS [30] [see Fig. 1(b)]. The WCS is obtained by strongly attenuating a beam derived from the same laser as the probe beam. The visibility of the HOM interference is calculated as

$$
V=1-\frac{p_{\text {ind }}}{p_{d}},
$$

where $p_{\text {ind }}\left(p_{d}\right)$ is the probability of a coincidence detection when the two photons are made indistinguishable (distinguishable). To generate the indistinguishable input, we match the polarization of the SP and the WCS and optimize the WCS waveform and the delay time with respect to the SP to maximize the overlap. For the distinguishable case, we apply a delay to the WCS pulse such that it is sent in a different time window than the single photon.

\section{HOM BETWEEN A SINGLE PHOTON AND A WEAK COHERENT STATE}

To estimate the indistinguishability of the Rydberg photons from the HOM visibility of Eq. (2), we follow the model presented in [20]. If three-photon coincidence events are negligible, the probability $p_{\text {ind }}$ can be computed as

$$
p_{\text {ind }}=p_{1}^{2} g^{(2)}+\frac{1}{4}|\bar{\alpha}|^{4}+(1-\eta) p_{1}|\bar{\alpha}|^{2},
$$

where the single-photon detection probability is $2 p_{1}$ and the two-photon detection probability is $p_{1}^{2} g^{(2)}$, while $|\bar{\alpha}|^{2}$ is the mean number of photons detected on the WCS and $\eta$ is the indistinguishability factor. For completely distinguishable fields $(\eta=0)$, we obtain

$$
p_{d}=p_{1}^{2} g^{(2)}+\frac{1}{4}|\bar{\alpha}|^{4}+p_{1}|\bar{\alpha}|^{2} .
$$

Therefore, we can express the visibility as

$$
V=1-\frac{p_{\text {ind }}}{p_{d}}=\frac{\eta p_{1}|\bar{\alpha}|^{2}}{p_{1}^{2} g^{(2)}+\frac{1}{4}|\bar{\alpha}|^{4}+p_{1}|\bar{\alpha}|^{2}} .
$$

From Eq. (5) we extract that the visibility is maximum when $\frac{|\bar{\alpha}|^{4}}{4}=p_{1}^{2} g^{(2)}$, i.e., when the probability to detect two photons coming from the WCS is the same as from the singlephoton source, leading to $V_{\max }=\frac{\eta}{\sqrt{g^{(2)}}+1}$. Therefore, even for perfect single-photon sources $\left(g^{(2)} \rightarrow 0\right)$, HOM visibilities close to one can be obtained only for $\eta=1$ and very weak WCS. Nevertheless, even for imperfect sources, one can infer a value of $\eta$ by measuring $V$ for different values of $|\bar{\alpha}|^{2}$ and by fitting the results with Eq. (5).

\section{RESULTS}

\section{A. Single-photon generation}

Figure 3(a) shows the results of the Hanbury-Brown and Twiss experiment between different experimental trials, for photons obtained via the OR scheme. The detection window for this measurement is fixed to $\Delta t=500 \mathrm{~ns}$, longer than the retrieved pulse duration. The normalized coincidences at the same trial give $g_{\Delta t}^{(2)}=0.265 \pm 0.005$. The clear antibunching feature is a proof of quantum behavior and single-photon emission. One can see bunching between subsequent trials 
(a)

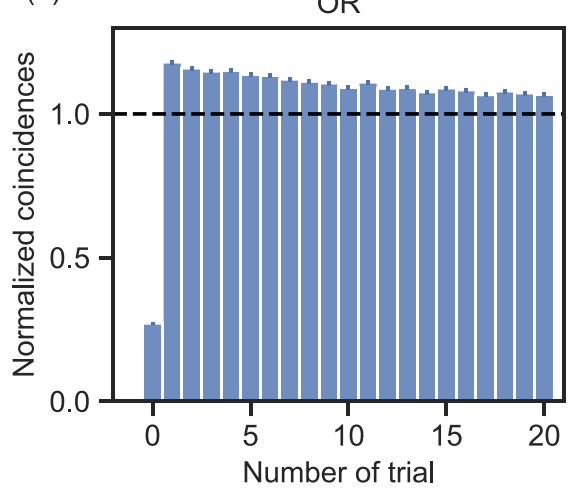

(b)

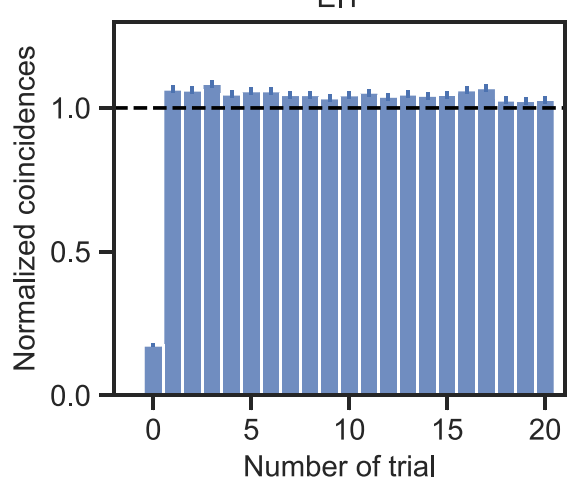

(c)

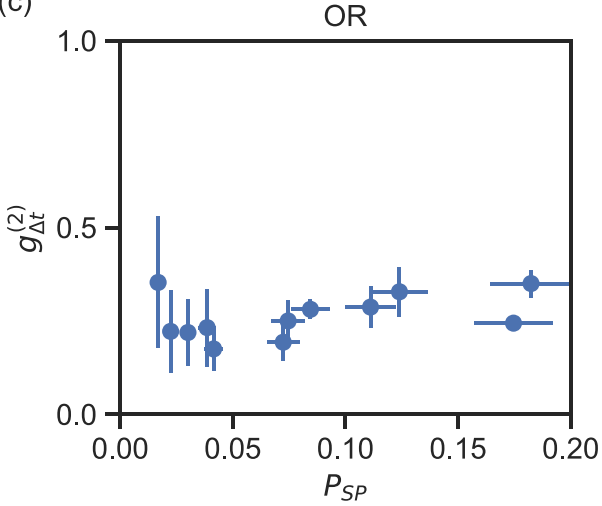

FIG. 3. Normalized coincidences between two different experimental trials, as a function of the number of trials between them, for (a) the OR excitation case and (b) the EIT case. (c) Variation of $g_{\Delta t}^{(2)}$ as a function of the probability of generating a single photon on the output $P_{\mathrm{SP}}$, for the OR case. Error bars correspond to one standard error. The behaviour with $P_{\mathrm{SP}}$ in the EIT case is shown in Fig. 8.

(normalized coincidences larger than 1), which can be explained by the creation of long-lived pollutants [26,31], which are nonretrievable Rydberg excitations that block the generation of retrievable Rydberg spin waves for several trials. These can be generated, for example, by the decay to other Rydberg states which are not coupled by the control field. Slightly better antibunching is observed for the EIT excitation scheme, with $g_{\Delta t}^{(2)}=0.165 \pm 0.005$ [see Fig. 3(b)].

The dependency of $g_{\Delta t}^{(2)}$ on the single-photon generation probability is shown in Fig. 3(c) for the OR case. The photon generation probability $P_{\mathrm{SP}}$ is defined as $P_{\mathrm{SP}}=\frac{p_{1}}{\epsilon_{\mathrm{det}}}$ where $\epsilon_{\mathrm{det}}=0.068 \pm 0.007$ is the detection efficiency, which includes all the transmission losses from the atomic ensemble to the first detector $(0.16 \pm 0.02)$ and the SPD efficiency $(0.43 \pm 0.04)$. Here we are assuming that the photons are emitted in the input mode, since we can study only the indistinguishability of in-coupled photons. $P_{\mathrm{SP}}$ is varied by controlling the mean number of photons in the probe pulse $n_{\text {in }}$. As one can see, the source preserves its quantum characteristic with increasing number of photons with $g_{\Delta t}^{(2)}<0.4$ for all performed measurements (with a maximal value of $0.35 \pm 0.04$ for $P_{\mathrm{SP}}=0.18 \pm 0.02$ ). The behavior of photons generated by EIT with increasing $n_{\text {in }}$ is very different and will be discussed in Sec. IV C.

\section{B. Photon indistinguishability}

\section{Single photon obtained with OR scheme}

We first analyze the case of excitation in the OR condition. In Fig. 4(a) we show an example of the pulses when they are made indistinguishable (top panel) and distinguishable (bottom). In the latter, the SP and the WCS are detected in well-separated time windows. From the distinguishable measurement, we extract the values of $|\bar{\alpha}|^{2}$ and $p_{1}$ by counting the photons arriving in the WCS and the SP windows, respectively, and the second-order correlation function of the single photons $g_{\Delta t}^{(2)}$ from the coincidences within the SP window [see Eq. (1)]. For the analysis of these data, we consider two different detection window durations: one with $\Delta t=500 \mathrm{ns,}$ which contains the full pulse, and another with $\Delta t=100 \mathrm{~ns}$, centered at the maximum amplitude.
In Fig. 4(b) we show the HOM visibility calculated from Eq. (2) as a function of $\frac{|\bar{\alpha}|^{2}}{2 p_{1}}$, which was varied by changing the mean number of photons in the WCS. In both cases, we achieve a visibility higher than the 0.5 , the classical value expected for interference of two WCS [32,33], attesting the quantum behavior of our source. The maximum visibility achieved is $0.66 \pm 0.07(0.58 \pm 0.03)$ for $\Delta t=100 \mathrm{~ns}(\Delta t=$ $500 \mathrm{~ns}$ ). Finally, we fit Eq. (5) to the data to obtain the indistinguishability factor $\eta$ ( $\eta$ is the only free parameter in the fitting procedure, and $g_{\Delta t}^{(2)}$ is fixed and measured separately; see the previous paragraph). For the full pulse, we find $\eta=$ $0.887 \pm 0.017$ and for the $100 \mathrm{~ns}$ window $\eta=0.984 \pm 0.013$.

\section{Single photon obtained with EIT}

The indistinguishability of EIT photons is obtained following the same method as in the OR excitation scheme. Figure 5(a) shows the selected detection window of $\Delta t=$ $600 \mathrm{~ns}$, containing the whole pulse, and $\Delta t=100 \mathrm{~ns}$, also
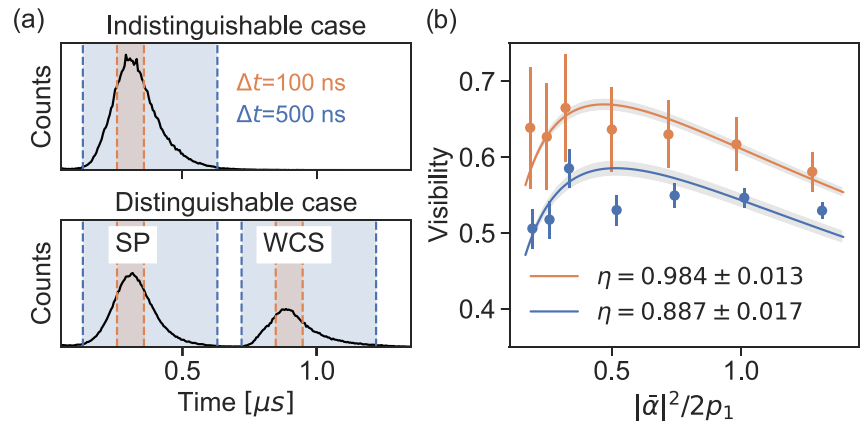

FIG. 4. Hong-Ou-Mandel measurements with photons generated with the OR scheme. (a) Temporal distribution of counts for the indistinguishable (top panel) and distinguishable (bottom panel) case. For the indistinguishable case, the single-photon (SP) and WCS pulse overlap in time, while for the distinguishable case, a delay between them is introduced. Two detection windows are selected with duration $\Delta t=500 \mathrm{~ns}$ (blue shaded region) and $\Delta t=100 \mathrm{~ns}$ (orange shaded region). (b) Visibility obtained by varying the mean number of photons in the WCS, for both observation windows. The shaded areas correspond to the error bars of $\eta$. 

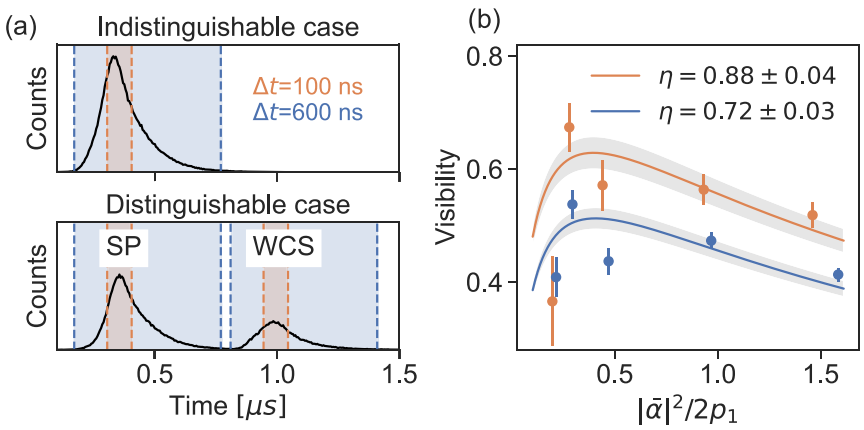

FIG. 5. Hong-Ou-Mandel measurements with photons generated with the EIT scheme. (a) Temporal distribution of counts for the indistinguishable (top panel) and distinguishable (bottom panel) case. (b) Visibility obtained by varying the mean number of photons in the WCS, for a time observation window of $\Delta t=600 \mathrm{~ns}$ (blue points) and $\Delta t=100 \mathrm{~ns}$ (red points). The shaded areas correspond to the error bars of $\eta$.

centered around the maximum. Note that the observation window of $\Delta t=600 \mathrm{~ns}$ is not centered at maximum amplitude, but optimized to include a larger portion of the pulse, because in contrast to the OR excitation, the pulse is less symmetric. Figure 5(b) shows the measurement of HOM visibility as a function of $\frac{|\bar{\alpha}|^{2}}{2 p_{1}}$. We observe that the visibility achieved is lower than for the OR case. For the measurement with a detection window of $\Delta t=600 \mathrm{~ns}$, it remains mostly below 0.5 , with a maximum value of $V=0.54 \pm 0.03$. For the smaller window of $\Delta t=100 \mathrm{~ns}$, it reaches values slightly over 0.5 with a maximum of $V=0.67 \pm 0.09$. Those visibility values lead to the indistinguishability factor $\eta=0.88 \pm 0.04(0.72 \pm$ 0.03 ) for $\Delta t=100(600) \mathrm{ns}$, significantly smaller than what we observe for the OR case [see Fig. 4(b)]. Note that for this experiment, there was a small frequency shift of $380 \mathrm{kHz}$ between the WCS and the retrieved single photon (a posteriori realized experimental imperfection), which is expected to cause a decrease of $3 \%$ in the indistinguishability, and therefore, it is not enough to explain the mismatch. Even though the HOM visibilities are lower for the single photons generated under EIT conditions, the values of $g_{\Delta t}^{(2)}$ are better than in the OR excitation case, as presented in Sec. IV A.

\section{Time-resolved HOM measurements}

It is relevant to mention that reducing the window of observation leads to higher visibilities and higher indistinguishability factors, which are in accordance with previous observations $[20,25]$. This is due to the fact that restricting the window of observation to values smaller than the coherence time of the retrieved photons can decrease the distinguishability between photons and therefore increase the visibility. This effect is investigated further in Fig. 6, for both the EIT and the OR case. Although the probability to generate a photon $P_{\mathrm{SP}}$ increases with increased observation window, the decrease in the indistinguishability factor and increase of the autocorrelation function is clear, implying a statistical decrease in the quality of the single photon. This decrease could be explained by the finite laser linewidths, which limits the coherence time of both the WCS and the single photon [34-36]. Another

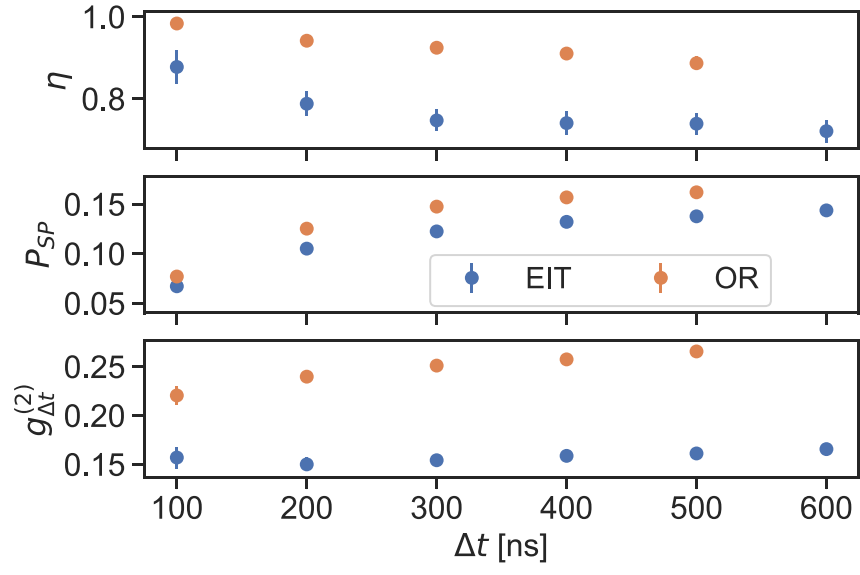

FIG. 6. Variation of the indistinguishability factor $\eta$ between the WCS and SP (top panel), variation of the generation probability $P_{\mathrm{SP}}$ of a single photon (second panel), and variation of the $g_{\Delta t}^{(2)}$ (bottom panel) as a function of the detection window $\Delta t$, for OR excitation and EIT conditions.

reason could be the temporal waveform mismatch between the single photons retrieved from the Rydberg ensemble and the WCS. However, we infer a temporal indistinguishability factor for the whole pulse above $98 \%$ for OR excitation and about $97 \%$ for the EIT case, showing that this contribution is small. If the frequency shifts between WCS and SP central frequency in the EIT case is considered, the overlap for the whole pulse decreases to $94 \%$. We point out that this decrease in the overlap should be lower for shorter detection windows.

Accounting for the imperfect balance $(47 / 53)$ of the BS [37] results in slightly higher indistinguishability factors that are well inside the error bars. We also did an evaluation of the accidental coincidences due to dark counts, and they do not have a detrimental impact in our observations, i.e., a background correction of the data leaves results inside the error bars of the noncorrected values. Therefore, neither correction of BS imbalance nor subtraction of background was performed in the data presented here.

To further evaluate how the indistinguishability varies inside the photon wave packet, we perform a time-resolved analysis, as shown in Fig. 7(a). We take a detection window of
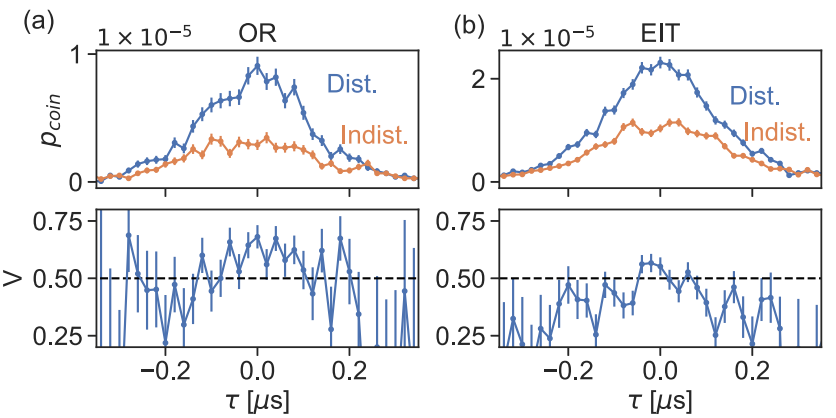

FIG. 7. Top: time-resolved coincidences for (a) OR excitation and (b) EIT excitation, for a bin size of $20 \mathrm{~ns}$, covering the whole extension of the pulse. Bottom: corresponding visibilities. For those measurements $|\bar{\alpha}|^{2} / 2 p_{1} \approx 0.5$ and the measured $g_{\Delta t}^{(2)}$ are $0.27 \pm 0.01$ for OR and $0.17 \pm 0.01$ for EIT. 
$10 \mathrm{~ns}$ inside the pulse and vary its position independently for both photodetectors to measure the coincidence profile with respect to the delay time of two-photon events. We observe that the visibility is higher around $\tau \approx 0$ and decreases on the wings, which confirms the measurements of Fig. 6. However, the decrease is small, and the visibility, and therefore the indistinguishability, remains high over the full single-photon pulse. This is in contrast to, e.g., single photons emitted by single trapped ions in cavities [38] or by most solid-state systems and shows that single photons emitted by Rydberg spin waves are well suited for quantum communication tasks, as shown previously [26]. Then the possibility of using an extended detection window results in increased efficiency in the generation of entanglement between remote nodes. The lower indistinguishability in EIT conditions is also confirmed by the time-resolved HOM measurement [see Fig. 7(b)], where we observe a similar behavior to the OR photons, but with lower visibility.

\section{Further investigation of the photon indistinguishability under EIT conditions}

We think that the significantly lower overlap $\eta$ for the EIT photons stems from the fact that the input photons can interact with the intermediate excited state. This effect was theoretically studied by Gorshkov et al. [39]. They concluded that for a fully blockaded medium under ideal Rydberg EIT conditions, the photon purity $P$ (which is an upper bound for the indistinguishability $\eta$ ) decreases monotonically from 1 to 0.5 with increasing $n_{\text {in }}$. In the ideal case, for a large number of input photons, only the first photon is mapped onto a Rydberg polariton, at the same time blocking within a blockade radius the transmission of subsequent photons, leading to photon scattering. The scattered photons carry information about the location of the Rydberg polariton, effectively lowering the purity of the polariton state.

To investigate the impact of the multiphoton scattering inside the probe pulse we performed the HOM measurements varying the number of input photons. The results are shown in Fig. 8. We observe that the indistinguishability $\eta$ lies between

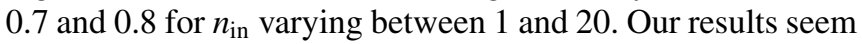
to contradict the model presented in [39]; however, our experiment does not fulfill some of the theory's assumptions. The model studies propagation of highly compressed slow light polaritons, while we perform a storage of much more weakly compressed pulses. Nevertheless, the experiment performed in Fig. 8 reaches a regime where strong scattering is present, as witnessed by a decrease in the transmission efficiency when observing pulse propagation under EIT conditions without storage [9]. Therefore, effects coming from the scatteringinduced decoherence should be present in our system.

The discrepancy can also be explained by the fact that Ref. [39] did not take into account the effect of filtering due to the finite-width EIT transparency window, which was later studied in [40]. The photon-scattering events project the Rydberg polariton onto a mixture of localized spin waves shorter than the blockade radius, effectively broadening its frequency spectrum. For larger input photon number the broadening is larger, and the spectrum can potentially exceed the EIT transparency linewidth. This effect leads to a frequency filtering

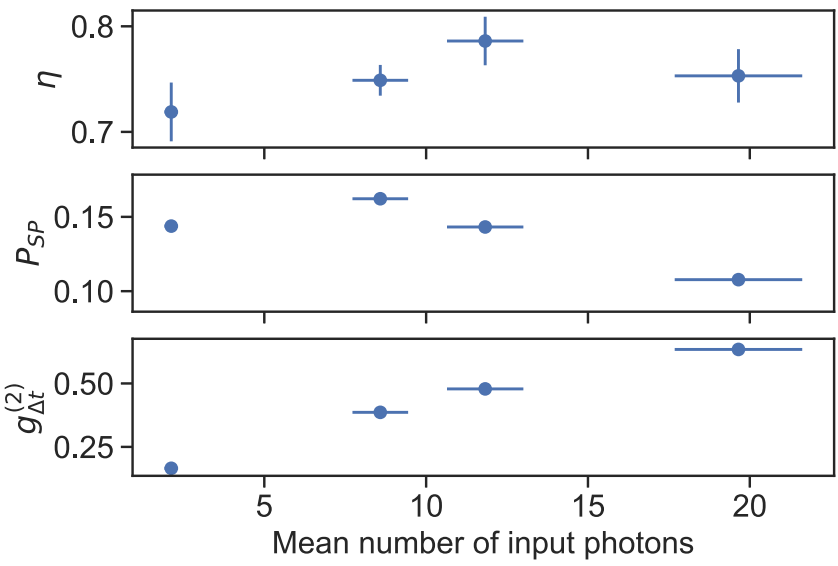

FIG. 8. Results of the HOM measurement under EIT conditions as a function of the mean number of input photons. Top pannel: Variation of the indistinguishability factor $\eta$ between the WCS and the emitted photon. For that measurement, there was a frequency shift of $320 \mathrm{kHz}$ between the WCS and the single photon. Middle pannel: Variation of the generation probability of a single photon $P_{\mathrm{SP}}$. Bottom panel: Variation of the second-order autocorrelation function $g_{\Delta t}^{(2)}$. Note that the error bars on the $x$-axis are mainly due to the error in detection efficiency, given by the systematic error of the power meter.

which could increase the indistinguishability of the transmitted photons and explain why $\eta$ does not drop with $n_{\text {in }}$.

In addition to the indistinguishability, we also measured the probability to generate a single photon $P_{\mathrm{SP}}$ as a function of $n_{\text {in }}$, as shown in Fig. 8(b). We observe that $P_{\mathrm{SP}}$ decreases with increasing numbers of input photons, particularly for $n_{\text {in }}>10$. This behavior is qualitatively consistent with the EIT filtering effect mentioned above. Another effect potentially causing the decrease of $P_{\mathrm{SP}}$ is the reabsorption of scattered photons [31] which can generate Rydberg excitations if they occur outside the blockaded region (one should remember that the coupling beam mode is significantly larger than the probe mode). These excitations form pollutants causing dephasing of the already existing excitation inhibiting its retrieval. One can also see excitations that are filtered out in the EIT filtering as pollutants: they block the medium and cannot be retrieved in the desired output mode. We expect both types of these pollutants to be short-lived, quickly removed by the control beam or the dipole trap. We comment on this later in this section.

The decrease in efficiency is accompanied by a strong degradation of the single-photon quality, witnessed by a large increase in $g_{\Delta t}^{(2)}$, up to $g_{\Delta t}^{(2)}=0.63 \pm 0.02$ for $n_{\text {in }} \approx 20$ [see Fig. 8(c)]. This degradation may be explained partly as an effect of pollutants. Detection of photons in a given trial is possible only if there is no pollutant in the medium, which happens with probability $\left(1-P_{p}\right)$, where $P_{p}$ is the probability of having a pollutant in the medium. One can observe that the denominator of Eq. (1) scales quadratically with the probability of not having a pollutant in a given trial [26] while the numerator scales only linearly. This results in an increase of $g_{\Delta t}^{(2)}$ of the retrieved photons by a factor $1 /\left(1-P_{p}\right)$. Another effect to consider is that the stored and retrieved pulse is longer than the blockade time $\tau_{b}=r_{b} / v_{g}$, such that when $n_{\text {in }}$ 
increases, there is an increasing probability of having more than one photon in the retrieved pulse.

To our knowledge, a proper theory describing the effect of pollutants on the indistinguishability and quality of single photons generated by Rydberg spin waves has not been proposed yet. One relevant open question is about the effect of the optical trap on pollutants. Since most Rydberg atoms experience an antitrap potential with the common dipole trap wavelengths used, we expect pollutants to experience a repulsive force each dipole trap cycle. The exact acceleration acquired depends heavily on $n$ [41]. Consequently, atoms capable of generating larger blockades should be expelled from the interaction region faster, while lower lying Rydberg atoms block a smaller region, but can stay longer in the interaction zone. A detailed study of this effect has not been performed yet to the best of our knowledge.

In summary, the study of the indistinguishability factor with the number of input photons could not fully explain the cause of the lower indistinguishability of the photons generated under EIT conditions, and therefore further studies should be carried out. For $n_{\text {in }} \approx 0.7$ (corresponding to the results of Fig. 5) we estimate $\sim 0.1$ photons within a blockade radius at a time, so the probability of a scattering event is already quite low. Therefore, we do not expect lower $n_{\text {in }}$ to give higher indistinguishability factors. Although a interesting next step would be to repeat this measurement in a regime where we do not observe filtering due to the narrow EIT transparency, this regime is extremely difficult to achieve experimentally [40]. Another possibility would be to study the behavior of the indistinguishability as a function of the detuning with respect to the intermediate excited state $\delta_{p}$, so that we could see how the characteristics of the emitted photons change when we move from the EIT conditions towards the OR excitation regime.

\section{CONCLUSION}

We conducted an extensive study of indistinguishability for single photons generated by cold ensembles of Rydberg atoms. We performed HOM interferometry between single photons and weak coherent states and compared two excitation schemes, OR and EIT storage, providing the figures of merit for the single-photon generation and characterization in each of those schemes.

We concluded that the OR scheme generates single photons with a higher degree of indistinguishability than those generated through stored Rydberg EIT, despite the fact that the latter exhibit a lower $g_{\Delta t}^{(2)}$. Moreover, the quality of the single photon generated under EIT conditions degrades faster with $n_{\text {in }}$. These effects may be due to the presence of scattering-induced decoherence and generation of pollutants. However, more measurements and a full model of decoherence in Rydberg EIT storage would be needed to quantify the consequences of the two effects.

For the OR scheme, we obtained a high degree of indistinguishability up to $89 \%$ for the full pulse, and up to 98\% of indistinguishability for a smaller detection window, in the case of OR excitation. We discussed the impact of extending the window of observation and concluded that, despite higher values of indistinguishability being achieved for smaller detection windows, the single photons remain highly indistinguishable for the whole duration of the pulse. These observations are specifically interesting for applications in quantum communications, where extending the window of observation implies a gain in efficiency and distant entanglement rate.

\section{ACKNOWLEDGMENTS}

The authors would like to thank Emanuele Distante for interesting discussions. This project received funding from the the Government of Spain (PID2019-106850RB-I00; Severo Ochoa CEX2019-000910-S), from the European Union's Horizon 2020 research and innovation program under Grant Agreement No. 899275 (DAALI), from the Gordon and Betty Moore Foundation through Grant No. GBMF7446 to H.d.R, from the Fundació Cellex and Fundació Mir-Puig, and from the Generalitat de Catalunya (CERCA, AGAUR).
[1] H. J. Kimble, The quantum internet, Nature (London) 453, 1023 (2008).

[2] M. Saffman, T. G. Walker, and K. Molmer, Quantum information with Rydberg atoms, Rev. Mod. Phys. 82, 2313 (2010).

[3] M. D. Lukin, M. Fleischhauer, R. Cote, L. M. Duan, D. Jaksch, J. I. Cirac, and P. Zoller, Dipole Blockade and Quantum Information Processing in Mesoscopic Atomic Ensembles, Phys. Rev. Lett. 87, 037901 (2001).

[4] A. V. Gorshkov, J. Otterbach, M. Fleischhauer, T. Pohl, and M. D. Lukin, Photon-Photon Interactions Via Rydberg Blockade, Phys. Rev. Lett. 107, 133602 (2011).

[5] D. Paredes-Barato and C. S. Adams, All-Optical Quantum Information Processing Using Rydberg Gates, Phys. Rev. Lett. 112, 040501 (2014).

[6] M. Khazali, K. Heshami, and C. Simon, Photon-photon gate via the interaction between two collective Rydberg excitations, Phys. Rev. A 91, 030301(R) (2015).
[7] A. K. Mohapatra, T. R. Jackson, and C. S. Adams, Coherent Optical Detection of Highly Excited Rydberg States Using Electromagnetically Induced Transparency, Phys. Rev. Lett. 98, 113003 (2007).

[8] J. D. Pritchard, K. J. Weatherill, and C. S. Adams, Nonlinear optics using cold Rydberg atoms, Annu. Rev. Cold At. Mol. 1, 301 (2013).

[9] T. Peyronel, O. Firstenberg, Q.-Y. Liang, S. Hofferberth, A. V. Gorshkov, T. Pohl, M. D. Lukin, and V. Vuletić, Quantum nonlinear optics with single photons enabled by strongly interacting atoms, Nature (London) 488, 57 (2012).

[10] D. Maxwell, D. J. Szwer, D. Paredes-Barato, H. Busche, J. D. Pritchard, A. Gauguet, K. J. Weatherill, M. P. A. Jones, and C. S. Adams, Storage and Control of Optical Photons Using Rydberg Polaritons, Phys. Rev. Lett. 110, 103001 (2013).

[11] S. Baur, D. Tiarks, G. Rempe, and S. Dürr, Single-Photon Switch Based on Rydberg Blockade, Phys. Rev. Lett. 112, 073901 (2014). 
[12] H. Gorniaczyk, C. Tresp, J. Schmidt, H. Fedder, and S. Hofferberth, Single-Photon Transistor Mediated by Interstate Rydberg Interactions, Phys. Rev. Lett. 113, 053601 (2014).

[13] D. Tiarks, S. Baur, K. Schneider, S. Dürr, and G. Rempe, Single-Photon Transistor Using a Förster Resonance, Phys. Rev. Lett. 113, 053602 (2014).

[14] D. Tiarks, S. Schmidt-Eberle, T. Stolz, G. Rempe, and S. Duerr, A photon-photon quantum gate based on Rydberg interactions, Nat. Phys. 15, 124 (2019).

[15] H. Busche, P. Huillery, S. W. Ball, T. Ilieva, M. P. A. Jones, and C. Adams, Contactless nonlinear optics mediated by long-range Rydberg interactions, Nat. Phys. 13, 655 (2017).

[16] Y. O. Dudin and A. Kuzmich, Strongly interacting Rydberg excitations of a cold atomic gas, Science 336, 887 (2012).

[17] F. Ripka, H. Kübler, R. Löw, and T. Pfau, A room-temperature single-photon source based on strongly interacting Rydberg atoms, Science 362, 446 (2018).

[18] J. Li, M.-T. Zhou, B. Jing, X.-J. Wang, S.-J. Yang, X. Jiang, K. Molmer, X.-H. Bao, and J.-W. Pan, Hong-Ou-Mandel Interference Between Two Deterministic Collective Excitations in an Atomic Ensemble, Phys. Rev. Lett. 117, 180501 (2016).

[19] L. Li and A. Kuzmich, Quantum memory with strong and controllable Rydberg-level interactions, Nat. Commun. 7, 13618 (2016).

[20] L. Li, Y. O. Dudin, and A. Kuzmich, Entanglement between light and an optical atomic excitation, Nature (London) 498, 466 (2013)

[21] J. Li, M.-T. Zhou, C.-W. Yang, P.-F. Sun, J.-L. Liu, X.-H. Bao, and J.-W. Pan, Semideterministic Entanglement Between a Single Photon and an Atomic Ensemble, Phys. Rev. Lett. 123, 140504 (2019).

[22] Y. Han, B. He, K. Heshami, C.-Z. Li, and C. Simon, Quantum repeaters based on Rydberg-blockade-coupled atomic ensembles, Phys. Rev. A 81, 052311 (2010).

[23] B. Zhao, M. Müller, K. Hammerer, and P. Zoller, Efficient quantum repeater based on deterministic Rydberg gates, Phys. Rev. A 81, 052329 (2010).

[24] C. K. Hong, Z. Y. Ou, and L. Mandel, Measurement of Subpicosecond Time Intervals Between Two Photons by Interference, Phys. Rev. Lett. 59, 2044 (1987).

[25] A. N. Craddock, J. Hannegan, D. P. Ornelas-Huerta, J. D. Siverns, A. J. Hachtel, E. A. Goldschmidt, J. V. Porto, Q. Quraishi, and S. L. Rolston, Quantum Interference Between Photons from an Atomic Ensemble and a Remote Atomic Ion, Phys. Rev. Lett. 123, 213601 (2019).

[26] D. P. Ornelas-Huerta, A. N. Craddock, E. A. Goldschmidt, A. J. Hachtel, Y. Wang, P. Bienias, A. V. Gorshkov, S. L. Rolston, and J. V. Porto, On-demand indistinguishable single photons from an efficient and pure source based on a Rydberg ensemble, Optica 7, 813 (2020).
[27] F. Bariani, Y. O. Dudin, T. A. B. Kennedy, and A. Kuzmich, Dephasing of Multiparticle Rydberg Excitations for Fast Entanglement Generation, Phys. Rev. Lett. 108, 030501 (2012).

[28] M. Fleischhauer and M. D. Lukin, Quantum memory for photons: Dark-state polaritons, Phys. Rev. A 65, 022314 (2002).

[29] E. Distante, A. Padrón-Brito, M. Cristiani, D. Paredes-Barato, and H. de Riedmatten, Storage Enhanced Nonlinearities in a Cold Atomic Rydberg Ensemble, Phys. Rev. Lett. 117, 113001 (2016).

[30] J. G. Rarity, P. R. Tapster, and R. Loudon, Non-classical interference between independent sources, J. Opt. B 7, S171 (2005).

[31] P. Bienias, J. Douglas, A. Paris-Mandoki, P. Titum, I. Mirgorodskiy, C. Tresp, E. Zeuthen, M. J. Gullans, M. Manzoni, S. Hofferberth, D. Chang, and A. V. Gorshkov, Photon propagation through dissipative Rydberg media at large input rates, Phys. Rev. Res. 2, 033049 (2020).

[32] L. Mandel, Photon interference and correlation effects produced by independent quantum sources, Phys. Rev. A 28, 929 (1983).

[33] J. Jin, J. A. Slater, E. Saglamyurek, N. Sinclair, M. George, R. Ricken, D. Oblak, W. Sohler, and W. Tittel, Two-photon interference of weak coherent laser pulses recalled from separate solid-state quantum memories, Nat. Commun. 4, 2386 (2013).

[34] T. Legero, T. Wilk, A. Kuhn, and G. Rempe, Time-resolved two-photon quantum interference, Appl. Phys. B 77, 797 (2003).

[35] J. Beugnon, M. P. A. Jones, J. Dingjan, B. Darquié, G. Messin, A. Browaeys, and P. Grangier, Quantum interference between two single photons emitted by independently trapped atoms, Nature (London) 440, 779 (2006).

[36] D. Felinto, C. W. Chou, J. Laurat, E. W. Schomburg, H. de Riedmatten, and H. J. Kimble, Conditional control of the quantum states of remote atomic memories for quantum networking, Nat. Phys. 2, 844 (2006).

[37] R. Uppu, T. A. W. Wolterink, T. B. H. Tentrup, and P. W. H. Pinkse, Quantum optics of lossy asymmetric beam splitters, Opt. Express 24, 16440 (2016).

[38] M. Meraner, A. Mazloom, V. Krutyanskiy, V. Krcmarsky, J. Schupp, D. A. Fioretto, P. Sekatski, T. E. Northup, N. Sangouard, and B. P. Lanyon, Indistinguishable photons from a trapped-ion quantum network node, Phys. Rev. A 102, 052614 (2020).

[39] A. V. Gorshkov, R. Nath, and T. Pohl, Dissipative ManyBody Quantum Optics in Rydberg Media, Phys. Rev. Lett. 110, 153601 (2013).

[40] E. Zeuthen, M. J. Gullans, M. F. Maghrebi, and A. V. Gorshkov, Correlated Photon Dynamics in Dissipative Rydberg Media, Phys. Rev. Lett. 119, 043602 (2017).

[41] Z. Lai, S. Zhang, Q. Gou, and Y. Li, Polarizabilities of Rydberg states of $\mathrm{Rb}$ atoms with $n$ up to 140, Phys. Rev. A 98, 052503 (2018). 